\title{
Nonlinear effects in the energy loss of a slow dipole in a free-electron gas
}

\author{
M. Alducin, ${ }^{1}$ R. Díez Muiño, ${ }^{2}$ J. I. Juaristi, ${ }^{3}$ and P. M. Echenique ${ }^{2,3}$ \\ ${ }^{1}$ Departamento de Ingeniería Eléctrica, ETSII, UPV/EHU, Alda. Urquijo s.n., 48013 Bilbao, Spain \\ ${ }^{2}$ Donostia International Physics Center (DIPC), P. Manuel de Lardizabal 4, 20018 San Sebastián, Spain \\ ${ }^{3}$ Departamento de Física de Materiales and Centro Mixto CSIC-UPV/EHU, Facultad de Químicas UPV/EHU, Apartado 1072, \\ 20080 San Sebastián, Spain
}

(Received 30 July 2002; published 18 November 2002)

\begin{abstract}
We analyze beyond linear-response theory the energy loss of a slow dipole moving inside a free-electron gas. The energy loss is obtained from a nonlinear treatment of the scattering of electrons at the dipole-induced potential. This potential and the total electronic density are calculated with density-functional theory. We focus on the interference effects, i.e., the difference between the energy loss of a dipole and that of the isolated charges forming it. Comparison of our results to those obtained in linear-response theory shows that a nonlinear treatment of the screening is required to accurately describe the energy loss of slow dipoles.
\end{abstract}

DOI: 10.1103/PhysRevA.66.054901

PACS number(s): 34.50.Bw, 34.50.Dy, 79.20.Rf

The study of the electronic excitations created by a charged projectile moving inside a solid or close to a surface provides information on the electronic properties of both the projectile and the target, as well as on the interaction between them. The electronic energy deposited in the medium can be partially compensated by a loss in the projectile kinetic energy. For small perturbations $(Z / v \ll 1$, where $Z$ is the charge of the projectile and $v$ its velocity, both in atomic units), linear theory gives an accurate description of the medium response and of the projectile energy loss. However, there is a wide range of velocities and/or projectile charges for which linear theory fails and nonperturbative approaches are required [1-3].

In the case of metal targets, the free-electron gas model correctly describes the valence-band excitation spectrum. The energy loss of atomic projectiles in free-electron-like metals has been widely studied and the experimental measurements are accurately reproduced by theoretical calculations [2,4-7]. However, new questions arise when the moving projectile is not a single ion or atom, but a cluster of two or more atomic constituents [8]. Correlation of charges traveling together in matter has been primarily studied in the range of high velocities, for which linear theory is in reasonable agreement with available experimental data [9-12]. The energy loss of a fast dimer can be theoretically described as the sum of the energy loss of each atom of the dimer plus an interference term [13]. In the low-energy range, there are theoretical works that show that nonlinear screening effects $[14,15]$ as well as multiple scattering of the medium electrons in the dimer potential $[15,16]$ introduce significant modifications in the calculation of the energy loss.

In this work we focus our attention on the energy loss of polar particles in metals. More precisely, we calculate the energy loss of a slow dipole moving inside a free-electron gas. In this case, the competition between the electron repulsion from the neighborhood of the negative charge and the piling up of electronic charge in the vicinity of the positive charge makes the screening process a complex phenomenon. Therefore, we study the correlation effects that arise when two charges of opposite sign move with the same velocity. In this respect, Arista [17] has recently published an interesting study on this problem, making use of the dielectric formalism. Here, we investigate the importance of nonlinear effects in this particular system in the low-energy range. Although the main purpose of our work is to address a fundamental theoretical question, there are real systems for which our results may be of interest, such as the electronic excitations created in the scattering of slow polar molecules off metal surfaces, and the energy loss of channeled polar molecules in capillaries. As a first approximation, neutral light ions in an excited electronic state could also be described by finite dipoles, as was suggested in Ref. [18]. Application of our results to actual energy-loss measurements of polar molecules in solids is more problematic because molecular ionization and/or dissociation processes are likely to occur.

The finite dipole is defined by two point charges $q= \pm 1$ separated by a distance $d$ (atomic units are used throughout). The dipole is embedded in a free-electron gas, whose mean electronic density is $n_{0}=3 / 4 \pi r_{s}^{3}$, and it moves with a velocity $\boldsymbol{v}$ with a modulus much smaller than the Fermi velocity $v_{F}$. The angle between the velocity and the dipole axis (that will be considered as the $O Z$ axis in our geometry) is called $\alpha$.

The calculation of the energy loss follows the same procedure that was already used and explained in Refs. [15] and [19] for other axially symmetric systems, and is only summarized here. First of all, we use density-functional theory and the Kohn-Sham scheme to calculate the ground state of a static dipole embedded in a free-electron gas [19]. Afterwards, the energy transferred from the moving dipole to the electron gas is obtained from the linear momentum transferred to the gas. More precisely, the total energy lost by the dipole per unit path length-also known as stopping power $S$-is obtained as

$$
S=\frac{d E}{d R}=\frac{1}{4 \pi^{3}} \int_{\mathrm{SFS}} d^{3} \boldsymbol{k} k^{2} \hat{\boldsymbol{v}} \cdot \boldsymbol{\sigma}_{\mathrm{tr}}(\boldsymbol{k}),
$$

where $\boldsymbol{k}$ and $\boldsymbol{k}^{\prime}\left(k=k^{\prime}\right)$, respectively, are the initial and final momenta of a medium electron in an individual collision, and $\hat{\boldsymbol{v}}$ is a unitary vector along the direction of $\boldsymbol{v}$. The integration is performed over a shifted Fermi sphere. In the limit 
of very slow velocities, only electrons at the Fermi level contribute to the energy loss. The transport cross section $\boldsymbol{\sigma}_{\mathrm{tr}}(\boldsymbol{k})$ (a vector quantity for nonspherically symmetric systems) is calculated in terms of the scattering amplitude $f\left(\boldsymbol{k}, \boldsymbol{k}^{\prime}\right)$ :

$$
\boldsymbol{\sigma}_{\mathrm{tr}}(\boldsymbol{k})=\int d \Omega_{\boldsymbol{k}^{\prime}}\left(\hat{\boldsymbol{k}}-\hat{\boldsymbol{k}}^{\prime}\right)\left|f\left(\boldsymbol{k}, \boldsymbol{k}^{\prime}\right)\right|^{2},
$$

with $\hat{\boldsymbol{k}}$ and $\hat{\boldsymbol{k}}^{\prime}$ being unitary vectors in the $\boldsymbol{k}$ and $\boldsymbol{k}^{\prime}$ directions, respectively. The scattering amplitude $f\left(\boldsymbol{k}, \boldsymbol{k}^{\prime}\right)$ is obtained from the asymptotic behavior of the Kohn-Sham orbitals at the Fermi level [19]. The transport cross section can be projected into two components, $\sigma_{\mathrm{tr}}^{\|}\left(\boldsymbol{k}_{F}\right)$ and $\sigma_{\mathrm{tr}}^{\perp}\left(\boldsymbol{k}_{F}\right)$, parallel and perpendicular to the dimer axis, respectively. The stopping power for velocities much smaller than the Fermi velocity can thus be written as

$$
S(\alpha)=F_{\|} v \cos ^{2} \alpha+F_{\perp} v \sin ^{2} \alpha,
$$

with

$$
F_{\|}=-\frac{k_{F}^{4}}{4 \pi^{3}} \int d \Omega_{\boldsymbol{k}_{F}} \sigma_{\mathrm{tr}}^{\|}\left(\boldsymbol{k}_{F}\right) \cos \theta_{\boldsymbol{k}_{F}}
$$

and

$$
F_{\perp}=-\frac{k_{F}^{4}}{4 \pi^{3}} \int d \Omega_{k_{F}} \sigma_{\mathrm{tr}}^{\perp}\left(\boldsymbol{k}_{F}\right) \sin \theta_{\boldsymbol{k}_{F}} \cos \varphi_{\boldsymbol{k}_{F}}
$$

$F_{\|}\left(F_{\perp}\right)$ is the friction coefficient (i.e., the energy loss per unit of path length and per unit of velocity) for a dipole moving along a direction parallel (perpendicular) to its axis, and $\left(\theta_{k_{F}}, \varphi_{k_{F}}\right)$ are the angular coordinates of the linear momentum at the Fermi level $\boldsymbol{k}_{F}$.

The stopping power of the dipole $S(\alpha)$ for any fixed orientation $\alpha$ of the velocity is given by Eq. (3). If the velocity direction is unknown, the stopping power has to be averaged over angles, giving as a final result

$$
S=\left[\frac{1}{3} F_{\|}+\frac{2}{3} F_{\perp}\right] v .
$$

Next, we apply our nonlinear formalism to study the dependence of the stopping power and the interference effects on the intercharge distance $d$ and the electronic density of the medium. In order to understand these dependences, let us start analyzing the distribution of the electrons in the vicinity of the dipole. Figure 1 shows the induced electronic density $\Delta n(r)$ around the dipole of $q= \pm 1$ inside an electron gas of $r_{s}=2$. The internuclear distance is $d=1$ a.u. In Fig. 1(a), we represent the contour plot of $\Delta n(r)$, in which darker zones correspond to higher electronic densities. The metal electrons are accumulated close to the positive charge $q=+1$ and are repelled from the vicinity of the negative charge $q=-1$. The asymmetry between the accumulation of electrons around the proton and the repulsion of electrons from the antiproton is due to our nonlinear treatment of the screening. Whereas an external negative charge cannot repel (from any point in the jellium) more electrons than the initial ones,
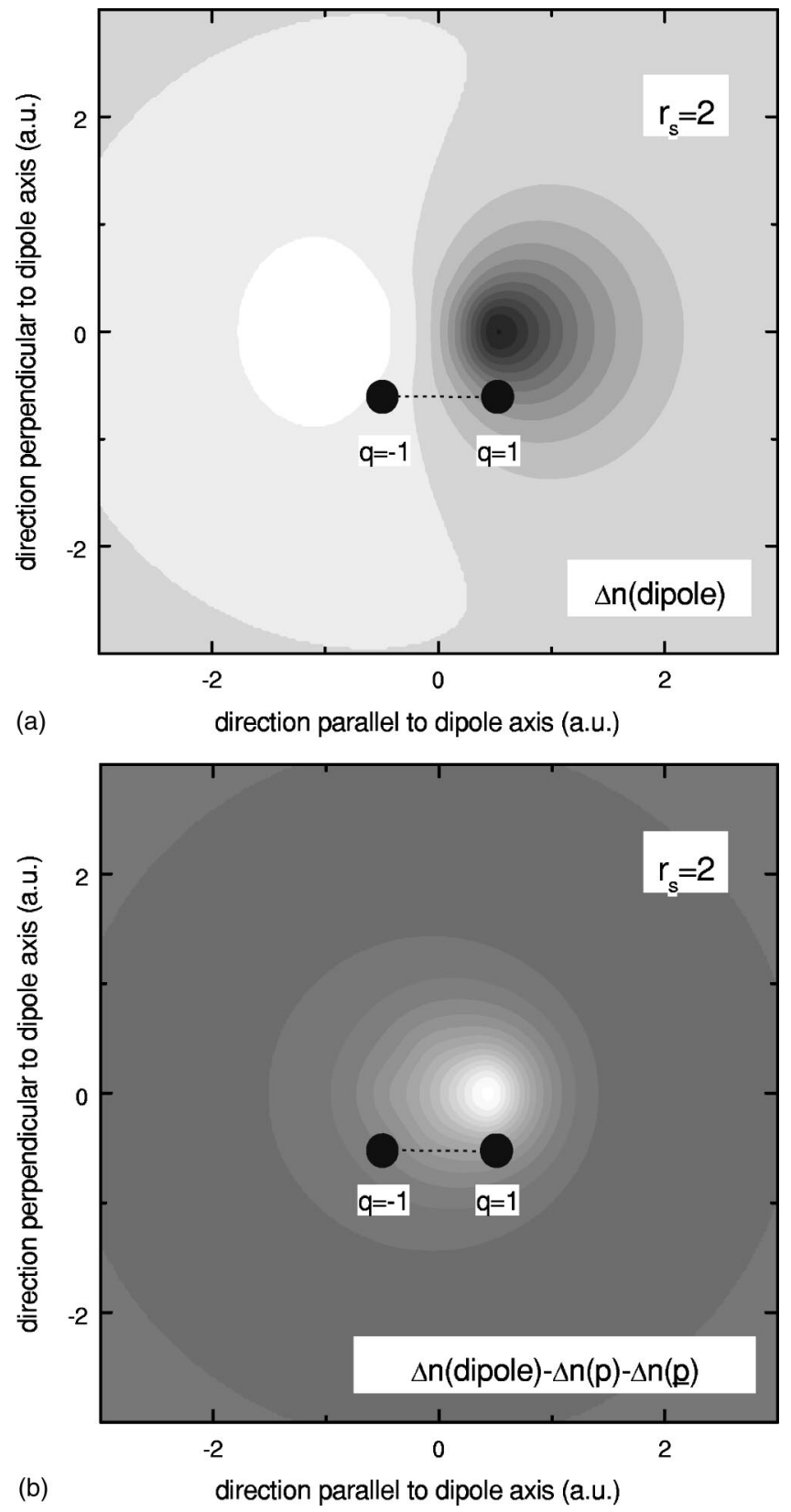

FIG. 1. DFT calculation of the electronic density $\Delta n(\mathbf{r})$ induced by a dipole in a free-electron gas of $r_{s}=2.0$. The charges of the dipole are $q= \pm 1$ and the distance between them is $d=1.0$ a.u. $\Delta n(\mathbf{r})$ is plotted in units of the background density $n_{0}$. (a) The upper panel shows a contour plot of $\Delta n(\mathbf{r})$ in a plane in which the dipole axis is contained. The lowest value of the induced density is $\Delta n(\mathbf{r}) / n_{0}=-1$. Darker zones (in steps of 0.5 ) correspond to higher values of $\Delta n(\mathbf{r})$. Abscissae represent distances in the direction parallel to the dipole axis, and ordinates represent distances in the direction perpendicular to it. All distances are in atomic units. (b) The lower panel shows the interference term in the induced electronic density of a dipole, i.e., $\Delta n$ (dipole $)-\Delta n(p)-\Delta n(\bar{p})$ in units of $n_{0}$. Darker zones (in steps of 0.5 ) correspond to higher values of the interference term. Lowest value of this difference is also -1 . Note that for clarity in the contour plots, the dipole is shifted downwards from its actual position. 


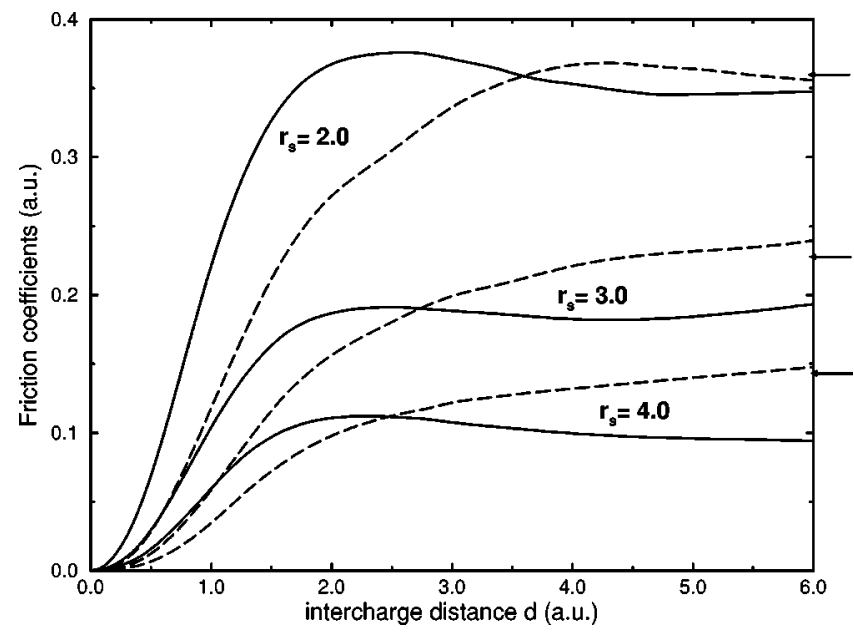

FIG. 2. DFT calculation of the friction coefficients of a slow dipole embedded in an electron gas as a function of the intercharge distance $d$. Results are shown for different electronic densities: $r_{s}$ $=2,3$, and 4 . Solid (dashed) lines correspond to the friction coefficient for a dipole moving parallel (perpendicular) to its axis. The arrows on the right ordinate axis indicate the asymptotic limit $F(p)+F(\bar{p})$ for each $r_{s}$ value (see text for explanation on this limit).

there is no restriction to attract as many as needed around an external positive charge. As a consequence, the screening length for the proton is shorter [3]. In the contour plot, this effect causes that the induced charge is more extended close to the antiproton than to the proton. We would like to remark that the scale chosen to plot $\Delta n(r)$ does not allow to observe the Friedel oscillations though they are present. The contour plot of Fig. 1(b) shows the interference term in the induced electronic density, $\Delta n$ (dipole) $-\Delta n(p)-\Delta n(\bar{p})$, where $\Delta n(p)$ and $\Delta n(\bar{p})$ are, respectively, the electronic densities induced by a proton and by an antiproton inmersed in the electron gas. The main effect of the interference is to reduce the induced electronic density near the positive charge.

In Fig. 2, the friction coefficients $F_{\|}$(solid lines) and $F_{\perp}$ (dotted lines) are represented as a function of the intercharge distance $d$ for three different electronic densities $\left(r_{s}\right.$ $=2,3,4) . F_{\|}$and $F_{\perp}$ approach zero in the limit $d \rightarrow 0$, since the finite dipole vanishes, i.e., there is no external perturbation. For large values of the intercharge distance, both $F_{\|}$and $F_{\perp}$ tend to the sum of the proton and antiproton friction coefficients, $F(p)+F(\bar{p})$. As $d$ increases, the interaction between both charges is totally screened by the electrons in the electron gas, and the dipole behaves as two independent charges moving in the medium. These limits $[F(p)+F(\bar{p})]$ are indicated by arrows in Fig. 2 for each electronic density. Since the screening is more effective for higher densities, the large-distance limit is reached faster as decreasing $r_{s} . F_{\|}$and $F_{\perp}$ have a nonmonotonic behavior with the distance $d$, which is essentially related to complex interference effects in the two-center potential. At small distances, $F_{\|}$dominates over $F_{\perp}$, whereas the opposite is true for large $d$. A remarkable feature is that the crossing distance between $F_{\|}$and $F_{\perp}$ goes towards smaller distances as $r_{s}$ increases, in contrast to what is obtained in a linear calcula-

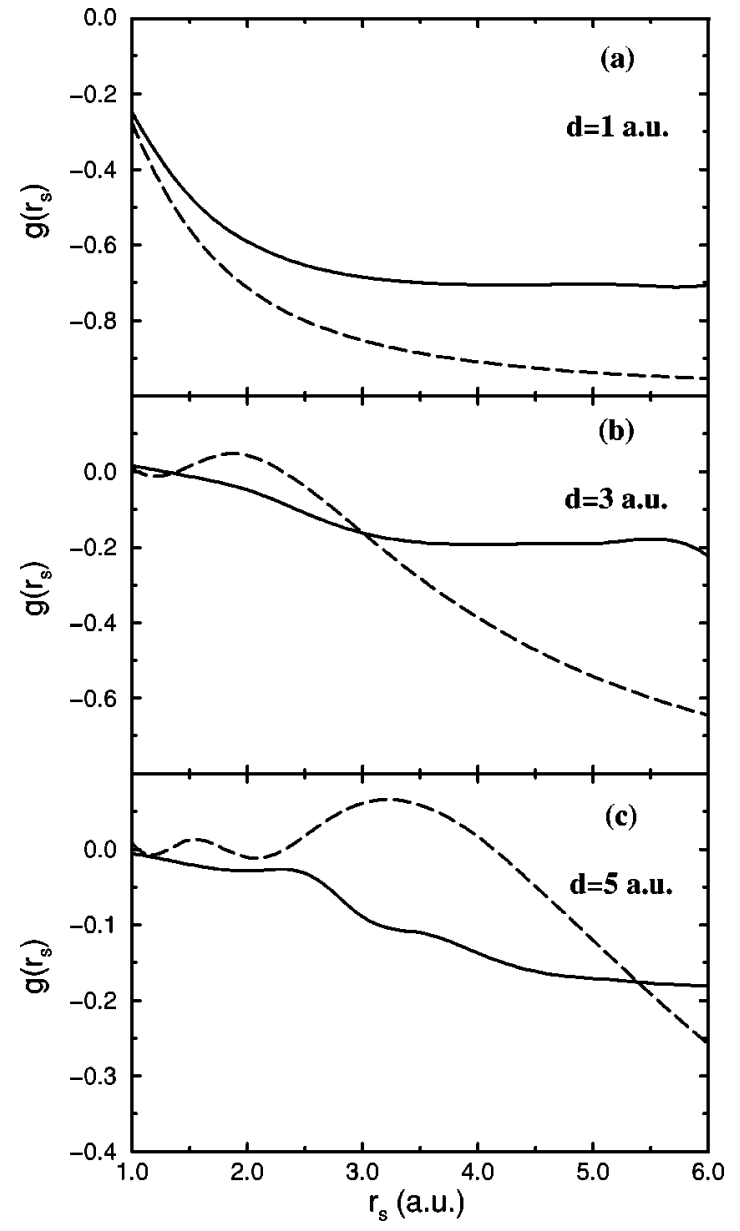

FIG. 3. Dependence of the vicinage effect measured by $g\left(r_{s}\right)$ [see Eq. (8)] on the jellium electronic density. The three panels show the results obtained for three different intercharge distances: (a) $d=1$ a.u., (b) $d=3$ a.u., and (c) $d=5$ a.u. Solid lines correspond to the DFT calculation and dashed lines to the LRT calculation.

tion. In case of $\mathrm{H}_{2}$ molecules, the crossing distance, obtained using both linear [16] and nonlinear [15] screening models, increases with $r_{s}$. Therefore, we conclude that the reduction of the crossing distance, with increasing $r_{s}$ is a genuine nonlinear effect that appears due to the different ways positive and negative charges are screened.

In the study of the energy loss of different molecules in solids, the so-called vicinage effect is one of the magnitudes of interest. In our case, the vicinage effect for a dipole is measured as the difference between the energy loss of the isolated charges $(p$ and $\bar{p})$ and that of the dipole itself. In order to analyze the relevance of our nonlinear treatment, we also calculate the vicinage effect for the dipole using linearresponse theory (LRT) and the dielectric formalism. The stopping power averaged over angles is given in this formalism by [17]

$$
\begin{aligned}
S_{L R T}(\text { dipole })= & \frac{1}{\pi^{2} v} \int \frac{d^{3} \mathbf{k}}{k^{2}}(\mathbf{k} \cdot \mathbf{v}) \\
& \times\left[1-\frac{\sin (k d)}{k d}\right] \operatorname{Im}\left[\frac{-1}{\epsilon(k, \mathbf{k} \cdot \mathbf{v})}\right],
\end{aligned}
$$


where $\epsilon(k, \omega)$ is the Lindhard dielectric function [20]. In the regime $v \ll v_{F}, S_{L R T}$ (dipole) is mainly ruled by the factor in brackets, $[1-\sin (k d) / k d]$, in which the first term arises from the stopping power of $p$ and $\bar{p}$, and the second one from the interference between both charges.

The vicinage effect for the dipole is measured in terms of the quantity

$$
g\left(r_{s}\right)=\frac{S(\text { dipole })}{S(p)+S(\bar{p})}-1 .
$$

According to this definition, $g\left(r_{s}\right) \rightarrow 0$ when the energy lost by the dipole approximates to the energy lost by $p$ and $\bar{p}$; whereas $g\left(r_{s}\right) \rightarrow-1$ if the dipole energy loss is negligible as compared to that of $p$ and $\bar{p}$. In Fig. 3, $g\left(r_{s}\right)$ is represented for three different distances: (a) $d=1$ a.u., (b) $d=3$ a.u., and (c) $d=5$ a.u. Solid lines show the results obtained with the density-functional-theory (DFT) calculation and dashed lines those with LRT. We find one common feature between both calculations. At high densities, since the screening around each charge is more effective, the dipole behaves as the two independent particles ( $p$ and $\bar{p})$; and, as a consequence, $g\left(r_{s}\right)$ approaches zero. Apart from this similarity, the vicinage effect predicted by both theories is remarkably different. At low densities, the DFT calculation of $g\left(r_{s}\right)$ exhibits a quite stable behavior on the electronic density. However, the LRT predicts a stronger decay of $g\left(r_{s}\right)$, as $r_{s}$ increases. This effect can be explained from the asymptotic behavior of the integral factor $[1-\sin (k d) / k d]$, which goes to zero in the limit $k_{F} d \ll 1$. The difference between both theories indicates that the dipole friction coefficient is underestimated when we use a linear description of the screening. The physical reason is that for increasing $r_{s}$, the wavelength of the electrons involved in the scattering process also increases $\left(\lambda_{F} \approx 3.3 r_{s}\right)$, thus the two-center character of the dipole potential is less important. More precisely, the potential experienced by the scattering electrons can be approximated by $V_{\text {dipole }}(r) \approx V_{p}(r)+V_{\bar{p}}^{-}(r), \lambda_{F} \gg d$. In DFT, the effective potentials of $p$ and $\bar{p}$ are quite different as a consequence of the nonlinear screening. However, since in LRT the effective potentials verify $V_{p}(r)=-V_{p}^{-}(r)$, the dipole potential $V_{\text {dipole }}(r)$ experienced by the scattering electrons is basically zero.

In summary, we have analyzed the nonlinear effects in the energy loss of a slow dipole moving inside an electron gas. Similar to the behavior of the friction coefficients in the $\mathrm{H}_{2}$ molecule, at small distances $F_{\|}$dominates over $F_{\perp}$ but as the distance increases the behavior is reversed. We find, however, a genuine nonlinear effect caused by the complex screening process of charges of opposite sign. Whereas in the $\mathrm{H}_{2}$ molecule, the crossing distance between $F_{\|}$and $F_{\perp}$ takes larger values as the electronic density decreases, in the dipole we just observe the opposite behavior. Finally, the calculation of the interference effects in the energy loss of a slow dipole also manifests the importance of a nonlinear treatment of the problem, particularly at low electronic densities. It is at such densities that the correlation effects of the two charges of opposite sign turn to be more important for the energy-loss process.

This work was supported in part by the Basque Departamento de Educación, Universidades e Investigación, the University of the Basque Country UPV/EHU (Grant No. 9/UPV 00206.215-13639/2001), and the Spanish Ministerio de Ciencia y Tecnología (Grant Nos. MAT2001-0946 and BFM2001-0076). R.D.M. acknowledges financial support by the Gipuzkoako Foru Aldundia.
[1] P. M. Echenique, F. Flores, and R. H. Ritchie, in Solid State Physics Series, edited by H. Ehrenreich and D. Turnbull (Academic, New York, 1990), Vol. 43, p. 230.

[2] I. Nagy, A. Arnau, and P.M. Echenique, Phys. Rev. A 40, 987 (1989).

[3] I. Nagy, A. Arnau, P.M. Echenique, and E. Zaremba, Phys. Rev. B 40, 11983 (1989).

[4] N. Wang and I. Nagy, Phys. Rev. A 55, 2083 (1997).

[5] A.F. Lifschitz and N.R. Arista, Phys. Rev. A 58, 2168 (1998).

[6] I. Nagy, Ch. Eppacher, and D. Semrad, Phys. Rev. B 62, 5260 (2000).

[7] A. Arnau, M. Peñalba, P.M. Echenique, F. Flores, and R.H. Ritchie, Phys. Rev. Lett. 65, 1024 (1990).

[8] N.R. Arista, Nucl. Instrum. Methods Phys. Res. B 164, 108 (2000).

[9] W. Brandt, A. Ratkowski, and R.H. Ritchie, Phys. Rev. Lett. 33, 1325 (1974).
[10] G. Basbas and R.H. Ritchie, Phys. Rev. A 25, 1943 (1982).

[11] F.J. Pérez-Pérez, I. Abril, R. García-Molina, and N.R. Arista, Phys. Rev. A 54, 4145 (1996).

[12] Z.L. Mišković, S.G. Davison, F.O. Goodman, W.-K. Liu, and Y.-N. Wang, Phys. Rev. A 63, 022901 (2001).

[13] N.R. Arista, Phys. Rev. B 18, 1 (1978).

[14] I. Nagy, A. Arnau, and P.M. Echenique, Nucl. Instrum. Methods Phys. Res. B 48, 54 (1990).

[15] R. Díez Muiño and A. Salin, Phys. Rev. B 62, 5207 (2000).

[16] H.M. Urbassek, V. Dröge, and R.M. Nieminen, J. Phys.: Condens. Matter 5, 3289 (1993).

[17] Néstor R. Arista, Phys. Rev. A 65, 022902 (2002).

[18] Néstor R. Arista, Phys. Rev. A 64, 032901 (2001).

[19] R. Díez Muiño and A. Salin, Phys. Rev. B 60, 2074 (1999).

[20] J. Lindhard and K. Dan, K. Dan. Vidensk. Selsk. Mat. Fys. Medd. 28, 1 (1954). 